\title{
Investigaciones en plantas con potencial bioactivo
}

\section{Investigations on plants with bioactive potential}

\author{
Catalina Rosales-López¹, Elizabeth Arnáez-Serrano², \\ lleana Moreira-González ${ }^{3}$, Giovanni Garro-Monge ${ }^{4}$, \\ Ana Laura Agüero-Hernández ${ }^{5}$, Karol Jiménez-Quesada ${ }^{6}$, \\ Ana Abdelnour-Esquivel ${ }^{7}$, Laura Calvo-Castro ${ }^{8}$
}

Rosales-López, C; Arnáez-Serrano, E; Moreira-González, I; Garro-Monge, G; Agüero-Hernández, A; Jiménez-Quesada, K; Abdelnour-Esquivel, A; Calvo-Castro, L. Investigaciones en plantas con potencial bioactivo. Tecnología en Marcha. Especial 2019. 25 Aniversario del Centro de Investigación en Biotecnología. Pág 12-21.

doi https://doi.org/10.18845/tm.v32i9.4621

1 Centro de Investigación en Biotecnología, Escuela de Biología, Instituto Tecnológico de Costa Rica, Costa Rica. Correo electrónico: crosales@itcr.ac.cr (iD) https://orcid.org/0000-0001-8336-0498

2 Centro de Investigación en Biotecnología, Escuela de Biología, Instituto Tecnológico de Costa Rica, Costa Rica. Correo electrónico: earnaez@itcr.ac.cr

(iD) https://orcid.org/0000-0003-4058-4429

3 Presidenta, Colegio de Biólogos de Costa Rica. Costa Rica. Correo electrónico: ilea2757@gmail.com (iD) https://orcid.org/0000-0001-9426-0986

4 Centro de Investigación en Biotecnología, Escuela de Biología, Instituto Tecnológico de Costa Rica, Costa Rica. Correo electrónico: ggarro@itcr.ac.cr (iD) https://orcid.org/0000-0001-7578-1938

5 Centro de Investigación en Biotecnología, Escuela de Biología, Instituto Tecnológico de Costa Rica, Costa Rica. Correo electrónico: anaguero@itcr.ac.cr (iD https://orcid.org/0000-0002-8538-2321

6 Centro de Investigación en Biotecnología, Escuela de Biología, Instituto Tecnológico de Costa Rica, Costa Rica. Correo electrónico: kjimenez@itcr.ac.cr (iD) https://orcid.org/0000-0002-0162-9279

7 Centro de Investigación en Biotecnología, Escuela de Biología, Instituto Tecnológico de Costa Rica, Costa Rica. Correo electrónico: aabdelnour@itcr.ac.cr (iD) https://orcid.org/0000-0002-8951-685

8 Centro de Investigación en Biotecnología, Escuela de Biología, Instituto Tecnológico de Costa Rica, Costa Rica. Correo electrónico: ancalvo@itcr.ac.cr 


\title{
Palabras clave
}

Plantas medicinales; compuestos bioactivos; metabolitos secundarios; fitoquímica.

\begin{abstract}
Resumen
El Centro de Investigación en Biotecnología (CIB) del Instituto Tecnológico de Costa Rica (TEC), cuenta con un grupo multidisciplinario de profesionales que orientan sus investigaciones en tres diferentes líneas de acción: Biotecnología Vegetal, Ambiental y Biomédica. En el área de Biotecnología Vegetal se desarrollan proyectos que incluyen investigaciones en productos naturales, metabolitos secundarios, compuestos antioxidantes, etnobotánica, bioprocesos, farmacognosia y cultivo de tejidos; todos bajo un mismo objetivo "el uso de recurso biótico como fuente de compuestos bioactivos con efecto positivo sobre la salud, que puedan ser utilizados como coadyuvante o fitofármaco natural para el hombre". En este artículo se presenta un breve resumen de los proyectos y logros que se han obtenido en esta área de investigación en el ClB.
\end{abstract}

\section{Keywords}

Medicinal plants; bioactive compounds; secondary metabolites; phytochemistry.

\begin{abstract}
The Biotechnology Research Center (CIB) at Costa Rica Institute of Technology (TEC) consists of a multidisciplinary group of researchers performing their research in three different lines of action in Biotechnology: Plants, Environmental and Biomedical Research. The research projects in Plant Biotechnology include investigations on plants with bioactive potential, natural products, secondary metabolites, antioxidant compounds, ethnobotany, bioprocesses, pharmacognosy and tissue culture. Researchers from different fields (biologists, agronomists and biotechnologists) collaborate in search of the same objective: "The use of biological resources as a source of secondary metabolites with a positive effect on health, which can be used as an adjuvant or for developing natural phytopharmaceuticals for humans". This article presents a brief summary of the projects and achievements that have been obtained in this area of research at CIB.
\end{abstract}

\section{Introducción}

El trabajo científico con plantas con potencial bioactivo es de vital importancia para la protección de la biodiversidad del país. El grave deterioro que han sufrido los productos no maderables de los bosques por su extracción indiscriminada, generó una fuerte tendencia a buscar sistemas de reproducción y manejo que garantizaran su uso comercial, sin amenazar la riqueza de los bosques. El Instituto Tecnológico de Costa Rica (TEC), inició el desarrollo de métodos alternativos de propagación de especies medicinales nativas desde 1988, con una investigación sobre la micropropagación de ipecacuana (la cual es considerara una planta medicinal por sus propiedades eméticas). Las investigaciones con ipecacuana continuaron en el 2007, enfocándose en el manejo agroecológico. Para el 2013 se realizó un perfil fitoquímico a la planta completa y, durante el periodo 2015-2017, se formuló un jarabe emético para animales domésticos, utilizando todos los órganos de la planta (Secreto industrial). En la actualidad, el CIB ha evaluado el potencial bioactivo de más de 20 plantas, desde la clasificación botánica hasta el análisis de su potencial anticancerígeno in vitro, y se están iniciando los primeros ensayos en modelos animales, con el fin de traducir estos esfuerzos a la práctica comercial. 


\section{Programa de Investigación y Extensión en Plantas Medicinales: políticas y lineamientos}

A partir del año 1997, un grupo de investigadores de la Escuela de Biología del TEC conformaron equipos de trabajo en el campo de las plantas medicinales. En el año 2000 se creó el "Programa de Investigación y Extensión en Plantas Medicinales, políticas y lineamientos", cuyo objetivo ha sido compartir los conocimientos adquiridos con el sector comercial nacional e internacional, dando especial atención al desarrollo de actividades que promuevan el uso sostenible de la biodiversidad, principalmente las plantas medicinales nativas o naturalizadas. Algunos de los ejes de conocimiento que se abarcaron con este Programa incluyen:

- Estudios biológicos (morfología, dinámica poblacional, reproducción, fenología, crecimiento y en general, biología de las especies).

- Propagación masiva y conservación de germoplasma.

- Aplicación de herramientas biotecnológicas (caracterización genética, estudios de actividades biológicas y principios activos).

- Estudios agronómicos y de mercadeo para producción y comercialización.

Unido a esto, en el año 2004 se creó el "Programa Nacional de Plantas Medicinales (PRONAPLAMED)", con el fin de conocer y potenciar los recursos de la biodiversidad de Costa Rica, adscrito a la Vicerrectoría de Investigación de la Universidad de Costa Rica y conformado por representantes de todas las universidades Estatales, miembros del Consejo Nacional de Producción (CNP), y la empresa privada (Laboratorios LISAN).

Debido a lo anterior, desde el año 2002 en el TEC se incrementaron las investigaciones en plantas con potencial bioactivo, sumando 28 proyectos de investigación desarrollados hasta la fecha (2019), logrando generar productos de interés comercial, numerosas publicaciones científicas, y la formación de alianzas con investigadores de diferentes instituciones (universidades y centros de investigación nacionales e internacionales, asociaciones y cooperativas de pequeños agricultores, y empresa privada).

\section{Investigaciones}

La mayoría de los proyectos realizados involucran a comunidades inmersas en ambientes con altas tasas de desempleo, subempleo, falta de acceso a conocimiento, maquinaria y equipo especializado, inexistencia de canales de comercialización, dependencia del monocultivo y la presión sobre la base de recursos naturales. Por lo tanto, muchos proyectos de investigación se han trabajado en conjunto con organizaciones y cooperativas, permitiendo la implementación de iniciativas para su desarrollo socioeconómico y productivo [1]. Con otras investigaciones, al estar vinculadas a la empresa privada, se ha logrado la futura comercialización del producto generado.

El interés por las investigaciones en plantas o microorganismos con compuestos bioactivos se debe a la gran biodiversidad de Costa Rica, donde la variedad de condiciones climáticas, zonas de cultivo, tipos de suelo y distintas variables bióticas, resultan en la existencia de variedades de especies locales con alto potencial fitoquímico. La mayoría de las investigaciones en este campo han buscado la obtención de extractos de plantas utilizando diversos solventes, técnicas de secado del material y extracción, así como la identificación fitoquímica de los grupos de metabolitos secundarios presentes, haciendo uso de técnicas cualitativas o equipos especializados. También se han realizado pruebas para la comprobación del potencial bioactivo 
en modelos in vitro e in vivo, con el uso de cultivos celulares y ensayos con modelos animales, enfocados en la búsqueda de propiedades con potencial en biomedicina.

Entre otros resultados, se han desarrollado tecnologías para la obtención de diversos productos fitoterapéuticos en forma de tinturas, jarabes, cremas, cosméticos, productos alimenticos y cápsulas, obtenidos a partir de extractos elaborados con el material vegetal. Además, se han utilizado las técnicas de cultivo vegetal in vitro con el objetivo de micropropagar el material vegetal, ya sea de toda la planta en estudio o partes de ella; por ejemplo, las raíces e incluso la obtención de un conjunto de células no diferenciadas denominada callo, con el fin de extraer los metabolitos secundarios de interés. Por otro lado, se han implementado técnicas moleculares y de ingeniería genética en algunas especies, con la intención de caracterizar genéticamente el material vegetal [2]. Se han obtenido resultados interesantes en estudios de metaboloma, logrando una mejor descripción de las rutas metabólicas, con el fin de poder modificarlas para aumentar la producción de los compuestos de interés [3]. La implementación de técnicas de ingeniería genética han permitido la obtención de cultivos de raíces pilosas, las cuales son un sistema muy promisorio para el escalamiento de la producción in vitro de metabolitos secundarios de importancia farmacológica [4], [5].

En el cuadro 1 se describen cada una de las especies de plantas con compuestos bioactivos estudiadas en el CIB, así como la marcha fitoquímica y pruebas de bioactividad realizadas. El CIB no solo ha centrado sus investigaciones en los metabolitos secundarios de las plantas, sino que también ha ampliado sus estudios con varios géneros de hongos que se consideran especies medicinales. Entre los hongos que se han investigado por sus compuestos bioactivos están: Rhodotorula sp y Pycnoporus sp (colorantes naturales) [35], Ganoderma sp (anticancerígeno y antiinflamatorio) (Tesis doctoral CRL, vigente), y Schizophyllum sp, Candida guillermondi y Ustilago sp (alérgenos) [36]. Por otro lado, también se ha estudiado el potencial nutricional y bioactivo de sustancias derivadas de microalgas, lo cual está descrito en otro artículo de esta misma edición.

Con el Laboratorio de Ingeniería de Tejidos del CIB cuenta con las facilidades para la caracterización de algunos efectos biológicos en modelos celulares y tisulares in vitro, de humanos y animales, incluyendo líneas celulares representativas de distintos tipos de cáncer en humanos, células de piel humana o piel humana reconstruida in vitro, modelos de músculo, hueso y células madre mesenquimales de roedores. Algunos de los efectos biológicos que se han evaluado en estos modelos incluyen: pruebas de citotoxicidad para la determinación de potencial anticancerígeno y de bioseguridad in vitro de sustancias; análisis de marcadores moleculares de diferenciación, apoptosis, necrosis o proliferación celular; y determinación del potencial regenerativo en modelos de piel. De esta forma, se han confirmado posibles aplicaciones biomédicas (preventivas y terapéuticas) de sustancias bioactivas de diversas plantas nativas de Costa Rica, incluyendo estudios publicados de mora [31] manzana, anona y ciruelo [20], [22] [23], guayaba [24] y varias plantas medicinales (Phyllanthus, Senna reticulata, Pettiveria alliaceae) [9], [23]. Actualmente, se desarrollan también ensayos de actividad biológica y toxicidad en modelos animales (roedores), en colaboración con otras universidades nacionales e internacionales. A futuro, se espera transferir estos resultados hacia la realización de pruebas clínicas y la generación de alimentos funcionales, nutracéuticos, y fitofármacos. 
Cuadro 1. Especies de plantas medicinales estudiadas por investigadores del Centro de Investigación en Biotecnología del TEC.

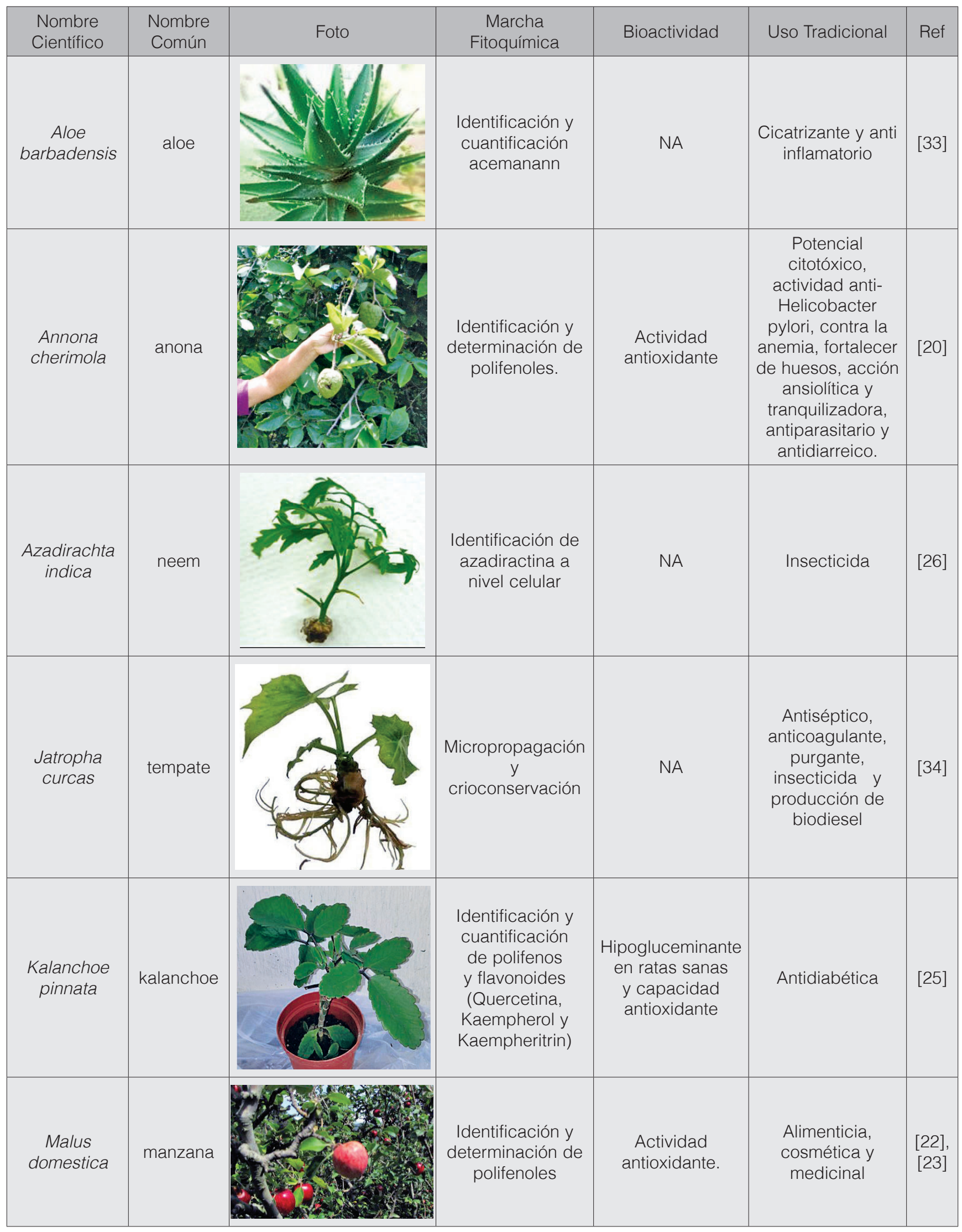




\begin{tabular}{|c|c|c|c|c|c|c|}
\hline $\begin{array}{l}\text { Nombre } \\
\text { Científico }\end{array}$ & $\begin{array}{l}\text { Nombre } \\
\text { Común }\end{array}$ & Foto & $\begin{array}{c}\text { Marcha } \\
\text { Fitoquímica }\end{array}$ & Bioactividad & Uso Tradicional & Ref \\
\hline $\begin{array}{c}\text { Moringa } \\
\text { oleifera }\end{array}$ & moringa & & NA & Citotoxicidad & $\begin{array}{l}\text { Fuente nutricional } \\
\text { por alta } \\
\text { concentración de } \\
\text { proteínas }\end{array}$ & {$[30]$} \\
\hline $\begin{array}{l}\text { Pettiveria } \\
\text { alliaceae }\end{array}$ & ajillo & & $\begin{array}{l}\text { Identificación y } \\
\text { determinación } \\
\text { de diferentes } \\
\text { compuestos } \\
\text { químicos. }\end{array}$ & NA & $\begin{array}{l}\text { Analgésico, } \\
\text { cardiovascular, } \\
\text { antiinflamatorio, } \\
\text { insecticida, } \\
\text { febrífugo, } \\
\text { gastrointestinal, } \\
\text { dolores de } \\
\text { muela, cabeza } \\
\text { y musculares, } \\
\text { enfermedades } \\
\text { de la piel y } \\
\text { antibacteriano, } \\
\text { entre otros. }\end{array}$ & $\begin{array}{l}{[9],} \\
{[10]}\end{array}$ \\
\hline $\begin{array}{l}\text { Phyllantus } \\
\text { acuminatus }\end{array}$ & chilillo & & $\begin{array}{c}\text { Identificación } \\
\text { de glicósidos: } \\
\text { filantostatinas en } \\
\text { plantas y cultivos } \\
\text { de raíces pilosas. } \\
\text { Identificación } \\
\text { de flavonoides y } \\
\text { otros }\end{array}$ & Anticancerígena & Antioxidante & $\begin{array}{l}{[9],} \\
{[10],} \\
{[11]} \\
{[12]} \\
{[13]} \\
{[5]}\end{array}$ \\
\hline $\begin{array}{l}\text { Phyllantus } \\
\text { niruri }\end{array}$ & $\begin{array}{l}\text { chanca } \\
\text { piedra }\end{array}$ & & $\begin{array}{l}\text { Establecimiento } \\
\text { de raíces pilosas. } \\
\text { Identificación } \\
\text { de flavonoides y } \\
\text { otros }\end{array}$ & NA & $\begin{array}{c}\text { Antiviral, } \\
\text { diurética, reduce } \\
\text { infecciones de } \\
\text { boca, garganta y } \\
\text { urinarias. }\end{array}$ & $\begin{array}{l}{[9],} \\
{[10]} \\
{[19]}\end{array}$ \\
\hline $\begin{array}{l}\text { Plantago } \\
\text { major }\end{array}$ & Ilantén & & $\begin{array}{l}\text { Identificación } \\
\text { de compuestos } \\
\text { verbascósidos } \\
\text { e iridoides: } \\
\text { aucubina, catalpol } \\
\text { en plantas y } \\
\text { líneas celulares }\end{array}$ & $\begin{array}{l}\text { Cicatrizante y } \\
\text { antimicrobiana }\end{array}$ & $\begin{array}{c}\text { Antioxidante, } \\
\text { antiinflamatorio, } \\
\text { antiespasmolítico }\end{array}$ & $\begin{array}{l}{[27],} \\
{[28]} \\
{[29]}\end{array}$ \\
\hline $\begin{array}{l}\text { Prunus } \\
\text { domestica }\end{array}$ & ciruelo & & $\begin{array}{l}\text { Identificación y } \\
\text { cuantificación de } \\
\text { polifenoles. }\end{array}$ & $\begin{array}{l}\text { Actividad } \\
\text { antioxidante }\end{array}$ & $\begin{array}{l}\text { Alimenticio, } \\
\text { cosmético y } \\
\text { medicinal. }\end{array}$ & $\begin{array}{l}{[20],} \\
{[21]} \\
{[22]}\end{array}$ \\
\hline
\end{tabular}




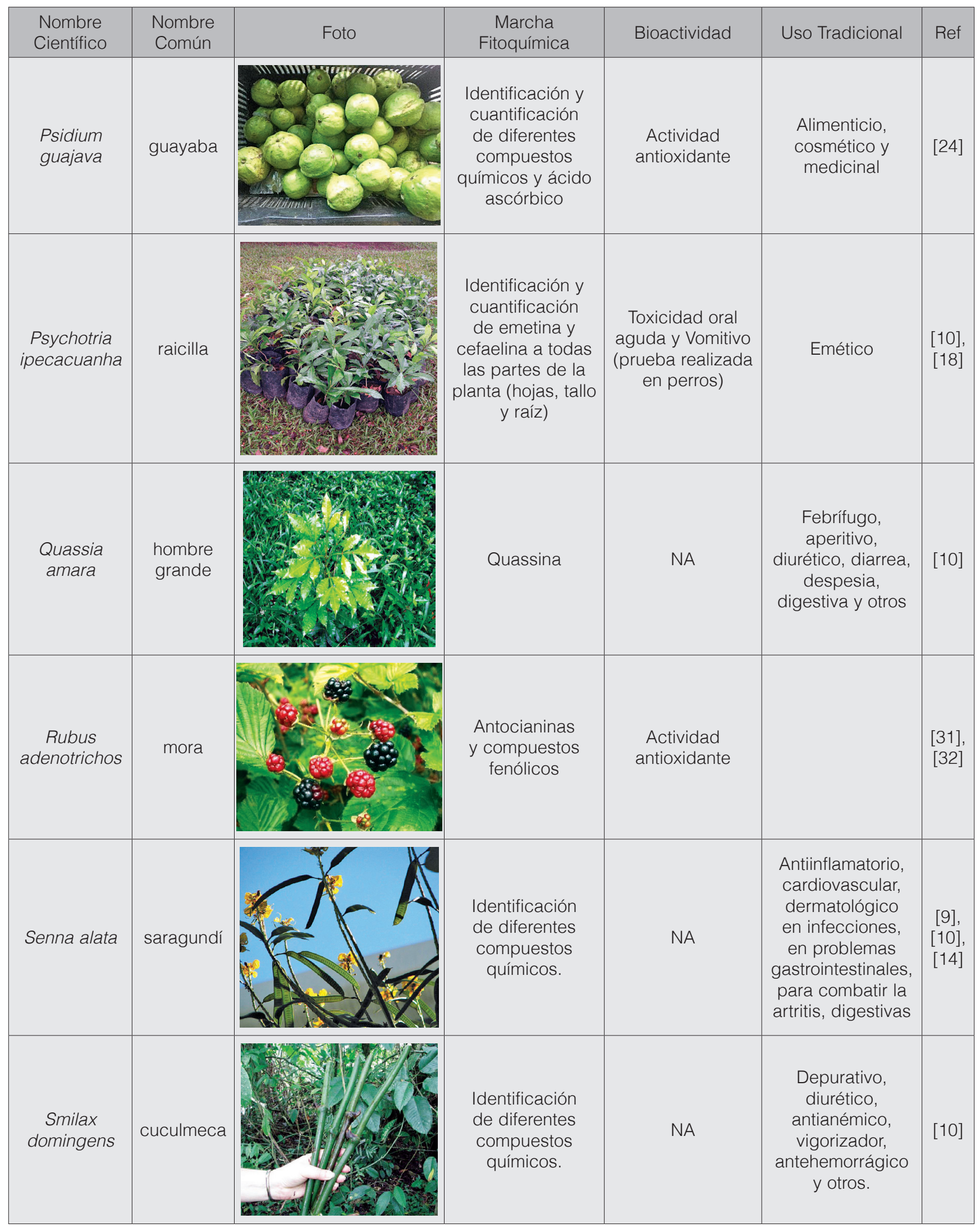




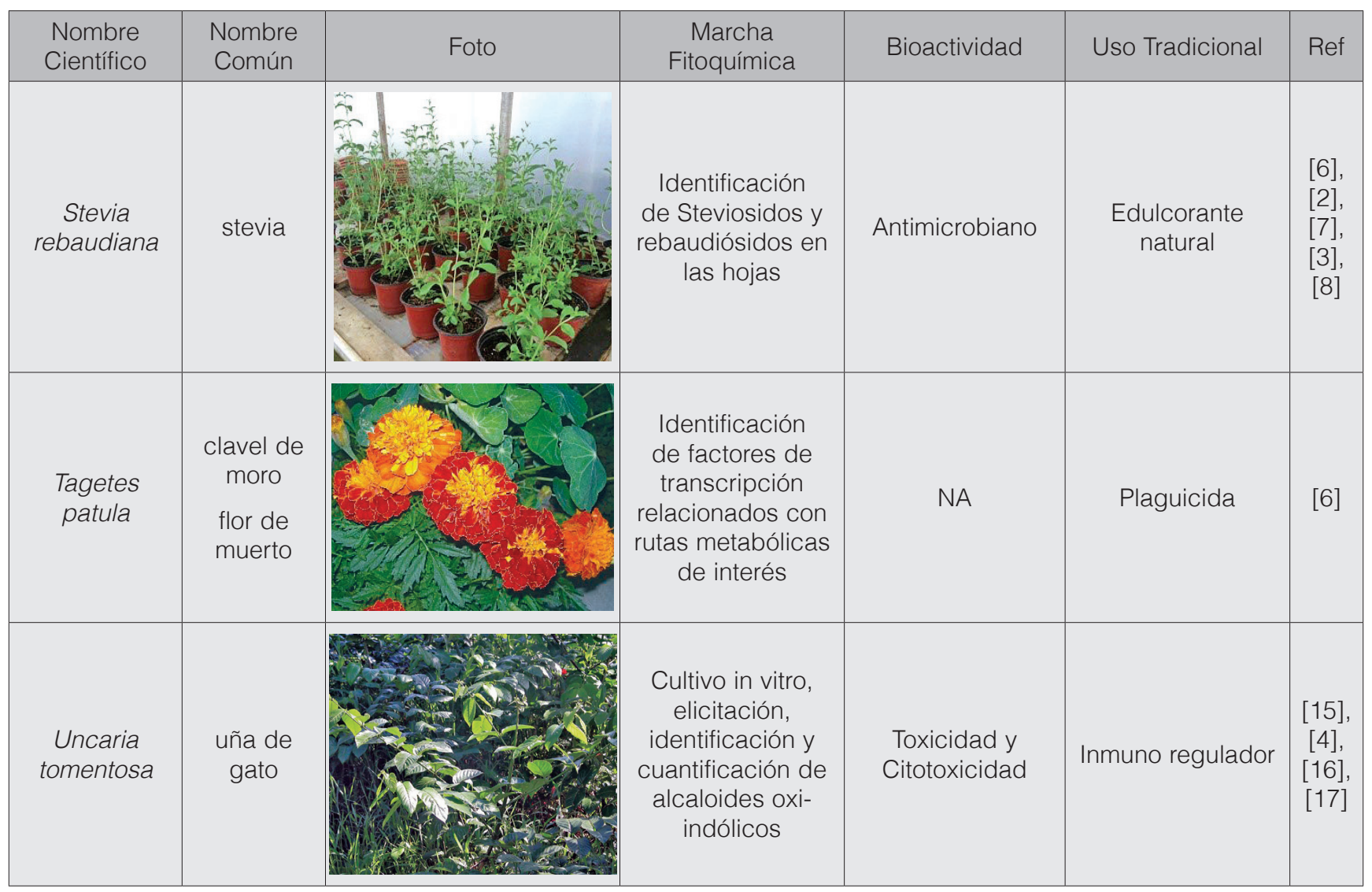

\section{Conclusión}

El Centro de Investigación en Biotecnología del Instituto Tecnológico de Costa Rica trabaja en asociación con la academia, entidades gubernamentales, instituciones públicas y privadas, y la sociedad en general, para seguir investigando los compuestos bioactivos de los recursos naturales nacionales. Investigadores del CIB unen esfuerzos y capacidades para colaborar con el posicionamiento de Costa Rica en el mapa mundial de las plantas y microorganismos con importancia médica. De esta forma, se promueve el desarrollo del país y el bienestar de su población con el estudio y producción de compuestos bioactivos que permitan la elaboración de medicamentos biotecnológicos.

\section{Referencias}

[1] I. Moreira y E. Arnáez, "Modelo de desarrollo económico local basado en alternativas de uso de plantas medicinales con componentes bioactivos". Biocenosis, vol. 25(1-2), pp. 42-45, 2012.

[2] G. Garro; K. Jiménez y S. Alvarenga, "Caracterización genética molecular de materiales procesados de Stevia rebaudiana utilizando la técnica de microsatélites”, Tecnología en Marcha, vol. 27 (3), pp. 32-40, 2014.

[3] K. Jiménez, A. Álvarez, G. Garro, "Identificación de secuencias génicas relacionadas con la ruta metabólica de síntesis de glicósidos en Stevia rebaudiana”. Tecnología en Marcha, vol 29(4), pp. 67-77, 2016.

[4] G. Garro, K. Ramijan, B. Blanco, K. Lam, S. Alvarenga, "Implementación de un protocolo para la producción de raíces pilosas (hairy roots) de uña de gato (Uncaria tomentosa) mediante transformación con Agrobacterium rhizogenes". Tecnología en Marcha, vol. 25 (3), pp. 28-38, 2012. 
[5] G. Garro-Monge, K. Jiménez-Quesada, y R. Pérez-Méndez, "Desarrollo de un sistema de cultivo in vitro de raíces pilosas de Phyllantus acumminatus, para la producción de compuestos con actividad biológica". Revista Tecnología en Marcha, vol. 31(3), pp. 66-73, 2018.

[6] K. Jiménez-Quesada, "Identificación de factores de transcripción putativos en Stevia rebaudiana y Tagetes ratula como herramienta para posterior uso en la descripción de rutas metabólicos de interés", Tesis de grado bachillerato, ITCR-2014.

[7] S. Alvarenga Venutolo, "Micropropagación masiva de Stevia rebaudiana Bertoni en sistemas de inmersión temporal". Cultivos Tropicales, vol. 36(3), pp. 50-57, 2015.

[8] C. Rosales, J. Brenes, K. Salas, S. Arce-Solano and A. Abdelnour-Esquivel, "Micropropagation of Stevia rebaudiana in temporary immersion systems as an alternative horticultural production method". Revista Chapingo Serie Horticultura, vol. 24 (1), pp. 69-84, 2018.

[9] I. Moreira, E. Arnáez, R. Murillo, S. Quesada, V. Castro, W. Zamora, M. Cordero, J. Loaiza y M. Navarro, "Estudio de cuatro plantas con uso medicinal tradicional cultivadas en las regiones Huetar Norte y Atlántica de Costa Rica". Revista Tecnología en Marcha, vol. 27 (4), pp. 70-77, 2014.

[10] E. Arnáez, I. Moreira, M. Navarro, "Manual descriptivo de nueve especies de plantas con potencial biactivo y su manejo agroecológico". Editorial Flaxo, 2016, pp 84.

[11] M. Navarro, I. Moreira, E. Arnaez, S. Quesada, "Flavonoids and Ellagitannins Characterization, Antioxidant and Cytotoxic Activities of Phyllanthus acuminatus Vahl." Plants, vol.6, pp.62, 2017.

[12] M.A. Sequeira-Obando, L.A. Calvo-Castro, I. Moreira-González, E. Arnáez-Serrano, M. Navarro, "Cytotoxic effect of an ethanol extract of Phyllanthus accuminatus leaves on human epithelial cancer cells". Pharmacology Online, vol.12, pp. 92, 2014.

[13] G. Garro-Monge, K. Jiménez-Quesada, L. Pérez-Méndez, "Desarrollo de un sistema de cultivo in vitro de raíces pilosas de Phyllantus acumminatus, para la producción de compuestos con actividad biológica". Revista Tecnología en Marcha, vol.31 (3), pp. 66-73, 2017.

[14] M. Navarro, I. Moreira, E. Arnaez, S. Quesada, G. Azofeifa, D. Alvarado and M. J. Monagas, "Proanthocyanidin Characterization, Antioxidant and Cytotoxic Activities of Three Plants Commonly Used in Traditional Medicine in Costa Rica: Petiveria alliaceae L., Phyllanthus niruri L. and Senna alata Willd". Plants, vol. 6(4), pp.50, 2017. doi:10.3390/plants6040050.

[15] S. Alvarenga-Venutolo, "Establecimiento in vitro y cultivo de células de la uña de gato (Uncaria tomentosa) (Willd.)", Revista Tecnología en Marcha, vol. 23(5), pp.22-24, 2010.

[16] S. Alvarenga-Venutolo, C. Rosales-López, L. Sánchez-Chinchilla, R. Muñoz-Arrieta and F. Aguilar-Cascante, "Seasonality effect on the composition of oxindole alkaloids from distinct organs of Uncaria tomentosa from the Caribbean region of Costa Rica". Phytochemistry, vol. 151, pp. 26-31, 2018.

[17] M. Navarro-Hoyos, D. Alvarado-Corella, I. Moreira-Gonzalez, E. Arnaez-Serrano, and M. Monagas, "Polyphenolic composition and antioxidant activity of aqueous and ethanolic extracts from Uncaria tomentosa bark and leaves". Antioxidants, vol. 7(5), pp.65, 2018.

[18] C. Rosales-López, R. Muñoz, and A. Abdelnour-Esquivel, "Emetine and cephaeline content in plants of Psychotria ipecacuanha in Costa Rica". Submitted at Revista Colombiana de Química, 2019.

[19] M. Jiménez, S. Venutolo and E. A. Fonseca, "Establecimiento del protocolo de micropropagación para la planta medicinal Phyllanthus niruri L. (Euphorbiaceae)". Tecnología en Marcha, vol. 20(2), pp. 21-31, 2007.

[20] M. Navarro, I. Moreira, E. Arnáez, R. Murillo, S. Quesada, W. Zamora y M. Cordero, "Estudio preliminar del potencial bioactivo de la Annona cherimola (anona) y Prunus domestica (ciruelo) cultivadas en Costa Rica". Revista Tecnología en Marcha, vol. 27, pp. 37-44, 2014.

[21] M. Navarro, Y. Zhao, J. Sun, I. Moreira, "Chemical Profiling of Polyphenolic Compounds in the Fruit Skin of Prunus domestica Plums from Costa Rica". J. Res. Anal., vol. 3, pp. 42-51, 2017.

[22] M. Navarro, I. Moreira, E. Arnaez, S. Quesada, "Polyphenolic Characterization and Antioxidant Activity of Malus domestica and Prunus domestica Cultivars from Costa Rica". Foods, vol. 7, pp.15, 2018.

[23] M. Navarro-Hoyos, I. Moreira-González, E. Arnáez-Serrano, R. Murillo-Masís, "Estudio preliminar del contenido polifenólico y capacidad antioxidante de la especie Malus domestica cultivada en Costa Rica". Rev. Tecnología en Marcha, vol.30, pp.3-13 2016. 
[24] K. Sánchez-Zúñiga, S. Castro-Piedra, I. Moreira-González, E. Arnáez-Serrano, M. Navarro-Hoyos y F. VargasHuertas, "Evaluación de las propiedades citotóxicas de un extracto de frutos de guayaba (Psidium guajava Var. Tai-Kuo-Bar)". Revista Tecnología en Marcha, vol. 30(4), pp.150-156, 2017. DOI: https://doi.org/10.18845/ tm.v30i4.3424

[25] A. Agüero-Hernández, C. Rosales-López, C. Herrera, A. Vargas-Picado, R. Muñoz, and A. Abdelnour-Esquivel, "Antidiabetic effect of Kalanchoe pinnata leaf extract". Submitted at Journal of Ethnopharmacology, 2019.

[26] K. Mata, "Optimización del cultivo en suspensión de Azadirachta indica A. Juss con miras al escalamiento en biorreactor". Tesis de Bachillerato, Ingeniería en Biotecnología, ITCR. 2014.

[27] B. Blanco, A. Saborío y G. Garro, "Descripción Anatómica, Propiedades Medicinales y Uso Potencial de Plantago major (llantén mayor)". Tecnología en Marcha, vol. 21 (2) pp.17-24, 2008.

[28] G. Garro y S. Alvarenga, "Optimización de un protocolo para el cultivo in vitro y la micropropagación masiva del Ilantén (Plantago major)". Tecnología en Marcha, vol. 22 (3), pp.25-33, 2009.

[29] K. Jiménez-Quesada y G. Garro-Monge, "Establecimiento de callogénesis somática en Plantago major e identificación de compuestos con actividad biológica". Revista Tecnología en Marcha, vol. 30(1), pp.-38, 2017.

[30] K. Sánchez-Zúñiga, L.A. Calvo-Castro, I. Moreira-González, M.A. Sequeira-Obando, E. Arnáez-Serrano and M. Navarro, "Preliminary evaluation of the cytotoxic capacity of an ethanol extract from Moringa oleifera leaves". Pharmacology, vol.12, pp.103, 2014.

[31] L. Calvo-Castro, D.N. Syed, J.C. Chamcheu and F. Vilela, "Protective effect of tropical highland blackberry juice (Rubus adenotrichos Schltdl.) against UVB-mediated damage in human epidermal keratinocytes and in a reconstituted skin equivalent model". Photochem. Photobiol., vol. 89, pp.1199-1207, 2013.

[32] A. Schmidt-Durán, C. Alvarado-Ulloa, R. Chacón-Cerdas, L. Alvarado-Marchena, and D. Flores-Mora, "Callogenesis and cell suspension establishment of tropical highland blackberry (Rubus adenotrichos Schltdl.) and its microscopic analysis". SpringerPlus, vol. 5(1), pp.1717, 2016.

[33] G. Garro-Monge, A. Gatica-Arias and M. Valdez-Melara, "Somatic embryogenesis, plant regeneration and Acemannan detection in Aloe (Aloe barbadensis, Mill)". Agronomía Costarricense, vol. 32(2), pp. 41-52, 2008.

[34] J. A. Prada, M. E. Aguilar, A. Abdelnour-Esquivel, and F. Engelmann, Cryopreservation of seeds and embryos of Jatropha curcas L. American Journal of Plant Sciences, vol. 6(01), pp. 172, 2015.

[35] C. Rosales-López, "Other important use of mushrooms". Revista Tecnología en Marcha, vol. 32(2), pp. 140-142, 2018.

[36] C. Rosales-López, K. Valerín-Berrocal, y V. Jiménez-Bonilla, "Crecimiento dimórfico y caracterización molecular de Candida guillermondi aislado de Pannicum maximun". Revista Tecnología en Marcha, vol. 31(1), pp.121132, 2018. 